\title{
Synthesis and characterization of nickel ferrite magnetic nanoparticles by co- precipitation method
}

\begin{abstract}
Magnetic nickel ferrite (NiFe2O4) nanoparticles have been synthesized via co-precipitation method by varying the metal precursors ratio. Four different precursors ratio (Fe:Ni) are varied at 40:60, 50:50, 60:40 and 80:20. The size of the nanoparticles is found to increase with increasing iron $(\mathrm{Fe})$ content. In addition, the morphology of the particles are observed to change from spherical to a shape similar to a nanooctahedral particle when the Fe content in the initial precursors ratio increases. The X-ray Diffraction (XRD) patterns have proved the presence of nickel ferrite nanoparticles. The magnetic properties characterized by Vibrating Sample Magnetometer (VSM) at room temperature proved that the assynthesized nickel ferrite nanoparticles are ferromagnetic and the saturation magnetization (Ms) increases with the content of Fe in the sample.
\end{abstract}

Keyword: Co-precipitation; Magnetic nanoparticles; Nickel ferrite; Precursor ratio 